\title{
MEU CORPO, MINHA FORTALEZA: UMA RELAÇÃO ENTRE ANATOMIA HUMANA E SAÚDE
}

\author{
Hyorrana Priscila Pereira Pinto ${ }^{1}$ \\ Amauri Pierucci ${ }^{2}$
}

\begin{abstract}
RESUMO: Este texto trata-se de um relato elaborado a partir de um projeto de extensão, cujo objetivo foi apresentar a alunos do ensino fundamental de uma escola de DiamantinaMG os sistemas morfológicos do corpo humano, correlacionando-os a temas ligados à saúde, hábitos saudáveis e prevenção de doenças. Inicialmente, foi realizado um breve histórico a respeito da Anatomia Humana, bem como a conceituação de educação em saúde. Em seguida, aplicou-se um questionário específico aos sujeitos da pesquisa, o qual direcionou os conteúdos morfológicos que seriam abordados. Em seguida, foram desenvolvidas atividades teóricas e práticas com enfoque nos assuntos ora propostos, no âmbito do Laboratório de Anatomia da Universidade Federal dos Vales do Jequitinhonha e Mucuri (UFVJM), fornecendo aos participantes uma maior abordagem em ações educativas e preventivas pertinentes ao seu cotidiano. Observou-se que a atividade prática associada ao conteúdo teórico facilitou o processo de ensino-aprendizagem. Quando acrescido o conhecimento sobre danos e ações preventivas, juntamente ao da educação em saúde, minimizou-se a ideia de não vulnerabilidade que os adolescentes possuem. Ainda, espera-se que atividades educativas nesta fase do desenvolvimento dos alunos envolvidos na pesquisa possam trazer benefícios futuros a sua saúde.
\end{abstract}

PALAVRAS-CHAVE: Anatomia humana. Sistemas morfológicos. Saúde. Corpo humano.

\section{My body, my fortress: a relationship between anatomy and health}

\begin{abstract}
This project introduced to school students the human body morphologic's systems and correlated them with issues's health, healthy lifestyle and prevention of diseases, based on health education. Initially, there was made a brief history about the Human Anatomy, as well as the concept of health education. Then was applied a specific questionnaire to the research subjects, which directed what morphologic's contents would be explored. Soon after, were developed theoretical and practical activities with a focus on proposed issues, in the Human Anatomy's Laboratory of Federal University of Jequitinhonha and Mucuri's Valleys, providing a greater focus on educational and preventive actions relevant to the daily student. It was observed that the practical activity associated with the theoretical content facilitated the teaching-learning process. When putted damage and preventive actions knowledge together to health education, downplayed the teenagers idea of non-vulnerability that they have. Still, it is expected that educational activities in this subject's stage of development may bring future benefits to their health.
\end{abstract}

KEYWORDS: Human Anatomy. Morphologic's systems. Health. Human body.

\footnotetext{
${ }^{1}$ Mestranda em Neurofisiologia da Universidade de Minas Gerais (hyorranapp@gmail.com).

${ }^{2}$ Doutor em Biologia Celular e Estrutural pela Universidade Estadual de Campinas, professor adjunto do Departamento de Ciências Básicas da Universidade Federal dos Vales do Jequitinhonha e Mucuri (amauri_pierucci@hotmail.com). 


\section{INTRODUÇÃO}

Anatomia Humana é a ciência que estuda a estrutura macroscópica do corpo humano e que, durante séculos, desencadeou o interesse inato das pessoas em conhecer seu próprio corpo. A princípio, nossos antepassados pré-históricos descobriram os órgãos dos animais por meio das caçadas. Naquele tempo, eles já sabiam que os músculos funcionavam para locomoção e o esqueleto como proteção de órgãos (VAN DE GRAAF, 2003).

Já os gregos expressavam a anatomia dos seus corpos nas competições atléticas e nas artes (escultura). No Egito, a preparação dos mortos pela técnica da mumificação, demonstrou o quanto esses povos possuíam conhecimento anatômico aprofundado das estruturas orgânicas. No período Renascentista, notou-se que grandes anatomistas, como Leonardo da Vinci e Michelangelo Buonarroti, detalharam as estruturas orgânicas e figuras humanas com grande fidelidade em suas obras de arte (VAN DE GRAAF, 2003).

De fato, para que houvesse uma melhor compreensão da estrutura e da função do corpo humano, a dissecação de cadáveres humanos tornou-se necessária e tal prática perdura até os dias de hoje. Essa atividade, assim como o conhecimento dos sistemas orgânicos como base de estudo e compreensão do corpo humano, é de suma importância para a formação de profissionais da área de saúde.

O Ministério da Educação (MEC) propõe que durante a educação básica, os estudantes tenham um conhecimento prévio acerca do corpo humano. Adicionalmente, os Parâmetros Curriculares Nacionais (PCN) descrevem que para o desenvolvimento do Eixo Temático "Ser Humano e Saúde" há a necessidade de se trabalhar, entre outros, os seguintes conteúdos: estudo dos sistemas e órgãos, prevenção de doenças, preservação da saúde coletiva e individual, riscos a integridade pessoal e social, valorização de hábitos saudáveis, métodos de contracepção e prevenção a doenças sexualmente transmissíveis e gravidez indesejada (BRASIL, 2000).

Como visto, a temática abordada neste texto é obrigatória na disciplina de Ciências no Ensino Fundamental, porém, nem todos têm acesso a este conhecimento. Dessa forma, acredita-se que a visitação ao âmbito de um laboratório de Anatomia Humana e a visualização dos órgãos, sistemas e aparelhos com preparações específicas funcionariam como elementos facilitadores do ensino-aprendizagem, com consequente entendimento de nossa estrutura corporal e, indiretamente, conhecimento da fisiologia humana (MONTES; CARDOSO; SOUZA, 2005).

Neste sentido, foi realizado um projeto, no qual se pretendeu agregar o conhecimento morfológico à Educação em Saúde, que se justifica pelo fato de ser um campo de conhecimento e de prática, que, historicamente, ocupa-se em promover saúde e prevenir doenças. O projeto, ainda, integrar os vários saberes, entre eles o científico e o popular, possibilitando aos sujeitos envolvidos uma visão crítica a respeito do tema "anatomia humana", bem como, uma participação responsável e autônoma frente à saúde no cotidiano (GAZZINELLI; PENNA, 2006).

O projeto, desenvolvido pela UFVJM, envolveu 33 alunos, de ambos os sexos, com idade entre 
10 e 15 anos, que cursavam o $9^{\circ}$ ano do Ensino Fundamental de uma escola da rede privada de ensino de Diamantina-MG.

Considerando a importância do conhecimento do corpo humano e do conceito de saúde como um equilíbrio do organismo humano, em seu aspecto biopsicossocial, o qual é resultante de práticas saudáveis de vida a partir da prevenção de doenças e da promoção à saúde, torna-se fundamental que ações interdisciplinares possam ser desenvolvidas junto aos escolares (GOMES et al., 2002).

$\mathrm{Na}$ literatura sobre Anatomia Humana são poucos os autores que a correlacionam à valorização da saúde e à adoção de hábitos saudáveis. A maioria prioriza a correlação entre adolescentes e temas restritos a sexualidade (FREITAS; DIAS, 2010). Dessa forma, a relevância do projeto deve-se a abordagem de conhecimentos que tendem a contribuir para um maior entendimento do corpo humano pelos adolescentes, visando o autocuidado e a promoção da saúde.

É sabido, nesta fase do desenvolvimento, os adolescentes estão motivados a buscar novas fontes de conhecimento capazes de influenciar na formação de sua personalidade, comportamento e no entendimento das mudanças corporais e psicológicas(FERREIRA,2001; FAUSTINI etal,2003; SILVA et al, 2004). Dessa forma, acredita-se que as atividades desenvolvidas no projeto supriram a carência de recursos práticos no âmbito escolar, assim como propiciaram o enriquecimento no conhecimento do próprio corpo e a inclusão de hábitos saudáveis, visando promover a saúde e prevenir doenças.

\section{OBJETIVO}

O objetivo principal do projeto foi apresentar o corpo humano a adolescentes, com idades entre 10 e 15 anos, estudantes do ensino fundamental de Diamantina-MG, de forma a correlacionar esse conhecimento aos hábitos saudáveis, à valorização da saúde e à prevenção de doenças.

\section{METODOLOGIA}

O projeto $^{3}$ teve como instituição proponente a Universidade Federal dos Vales do Jequitinhonha e Mucuri e, como instituição coparticipante, o Colégio Diamantinense. As atividades transcorreram de março a outubro de 2011.

Para as atividades, foram utilizadas diferentes maneiras de enfoque do corpo humano. Para tanto, foram dispostas algumas coleções de peças anatômicas artificiais(Modelo3B, Germany), além de uma coleção de peças cadavéricas, as quais foram demonstradas e reunidas nos diferentes Sistemas Orgânicos.

Como dito, o projeto envolveu 33 estudantes, de ambos os sexos, que cursavam o $9^{\circ}$ ano do Ensino Fundamental, com idade entre 10 e 15 anos. O número de alunos atendeu à demanda apresentada pelo referido colégio. As etapas de realização consistiram no envio do Termo de

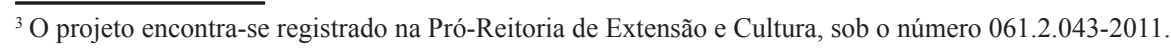


Consentimento Livre e Esclarecido (TCLE) aos pais ou responsáveis pelos estudantes, além da autorização da direção da escola no aceite do referido projeto, aplicação de um questionário específico (SANTANA; JORGE, 2007) aos alunos (Anexo I) e agendamento das visitas ao Laboratório de Anatomia Humana da UFVJM.

As atividades no Laboratório de Anatomia Humana foram realizadas em quatro dias por meio de oficinas (Anexo II). Todos os alunos participantes receberam Equipamentos de Proteção Individual (EPIs), entre eles, luvas de procedimento ambidestras e máscaras faciais, para proteção contra o formol. Vale salientar que caso houvesse qualquer risco para os adolescentes ou para outros sujeitos envolvidos, o projeto seria suspenso ou encerrado.

Ressalta-se que, para a realização das atividades, foram considerados os princípios da Educação em Saúde, que consiste em integrar os saberes científico e popular, possibilitando aos sujeitos envolvidos uma visão crítica e uma maior participação no processo de ensinoaprendizagem (GAZZINELLI; PENNA 2006). Assim, o tema foi apresentado aos adolescentes de maneira interativa, a partir do conhecimento teórico-prático, com o intuito de esclarecer as transformações ocorridas em seus próprios corpos. Tal fato possibilitou uma construção autônoma sobre os cuidados para consigo mesmos a partir da implantação da educação em saúde.

\section{RESULTADOS}

Após a aplicação do questionário específico e análise das respostas, percebeu-se que 63,63\% $(\mathrm{n}=21)$ dos entrevistados desconheciam o que era a ciência Anatomia Humana. Ainda, do total de alunos entrevistados $(100 \%), 78,78 \%(\mathrm{n}=26)$ nunca haviam adentrado a um Laboratório de Anatomia e 63,63\% ( $\mathrm{n}=21)$ nunca tinham observado um cadáver. Entretanto, $63,63 \%(\mathrm{n}=21)$ deles conseguiram correlacionar estrutura anatômica e seu próprio corpo, sendo que 69,69\% $(n=23)$ sabiam quais mudanças ocorrem em seu corpo durante o período da puberdade. Todos os entrevistados acreditam que é importante o conhecimento do corpo humano e que este projeto acrescentará algum tipo de conhecimento em sua vida.

Já quando perguntado aos adolescentes quais os sistemas que mais lhes interessavam, observouse que $6,06 \%(\mathrm{n}=02)$ têm interesse no Sistema Muscular, 6,06\% $(\mathrm{n}=02)$ preferem o Sistema Digestório, 9,09\% $(\mathrm{n}=03)$ têm curiosidade em aprofundar-se no estudo do Sistema Respiratório, $12,12 \%(\mathrm{n}=04)$ optaram pelo Sistema Reprodutor, $21,21 \%(\mathrm{n}=07)$ escolheram o Sistema Cardíaco e $24,24 \%(n=08)$ preferiram o Sistema Nervoso.

Após a análise quantitativa do questionário, deu-se início às atividades no Laboratório de Anatomia Humana da UFVJM. No primeiro dia, foi realizado um resumo teórico sobre a história da Anatomia Humana e a conceituação da Educação em Saúde. Posteriormente, foram exibidas figuras e fotos de diferentes partes do corpo humano, com o intuito de introduzir o que seria visualizado nas oficinas seguintes. Foram expostos, também, os principais objetivos do projeto: propiciar um maior conhecimento dos adolescentes a respeito do próprio corpo; despertá-los para a necessidade e a importância do autocuidado; auxiliá-los a tomar atitudes que tornem a 
fase adulta mais saudável; implantar a educação em saúde em seu cotidiano.

No decorrer da oficina, foram feitos alguns questionamentos pelos alunos: "Por que dessecar?"(A1); "Qual a diferença entre veias, artérias e nervos?" (A2); "Qual a real forma do coração?" (A3); "O que é o diafragma?" (A4); "Por que a parede do coração é maior de um lado?" (A5); “O sangue venoso é de coloração azul?" (A6); "A pele no formol fica de que cor?" (A7); “Onde se formam as pedras no rim?" (A8); "O que acontece no infarto?" (A9); "Por que os ossos são ocos?" (A10). Todas as perguntas foram respondidas resumidamente e a elas foi dado um maior enfoque nas aulas práticas.

No segundo dia, iniciaram-se os estudos com os sistemas ósseo, articular e muscular. Primariamente, foi conceituado o que é esqueleto, suas funções, morfologia e as variedades de substância óssea. Em seguida, foram conceituados as articulações e músculos, bem como, suas funções e classificações (DÂNGELO; FATTINI, 2002; SMELTZER; BARE, 2006).

No terceiro, foram abordados o sistema respiratório, o digestório e o cardíaco. Durante a apresentação do sistema respiratório foram estudadas todas as partes do mesmo, a função de cada item integrante, o conceito de hematose e o trajeto do ar no sistema, desde a entrada pelas narinas até a chegada aos pulmões (SMELTZER; BARE, 2006; DÂNGELO, FATTINI, 2002). Do sistema digestório, foram descritas suas características principais, como comprimento, localização, porções e funções, bem como a necessidade de suprimento constante de material nutritivo imprescindível para funcionalidade e vivacidade do organismo e o conceito de digestão (SMELTZER; BARE, 2006; DÂNGELO; FATTINI, 2002). Posteriormente, realizaram-se as atividades com respeito ao sistema cardiovascular, no qual foram descritas as funções básicas do sistema, a localização, morfologia, organização interna, partes do coração e os tipos de circulação, seguidas da sequência ao estudo dos vasos, no qual se abordou as principais características e diferenças entre os mesmos (DÂNGELO, FATTINI, 2002).

No quarto dia, foram expostos os sistemas urinário, reprodutor feminino e masculino e nervoso. Foram descritos os órgãos componentes de cada sistema, localização, características principais e funções correspondentes a cada órgão (CANALI; KRUEL, 2001; DÂNGELO; FATTINI, 2002; SMELTZER; BARE, 2006). Em relação ao sistema nervoso, foi retratada a classificação anatômica, citando-se os componentes pertinentes a cada porção, meninges, circulação do líquor, glândula hipófise e hormônios por ela produzidos (SMELTZER; BARE, 2006; DÂNGELO, FATTINI, 2002).

Para exposição e explicação dos sistemas foram utilizados todo o material anatômico e modelos sintéticos do acervo do Laboratório de Anatomia Humana da UFVJM, fundamentais para a abordagem dos conteúdos trabalhados nas oficinas.

\section{DISCUSSÃO}

A apresentação, com enfoque teórico-prático, das peças anatômicas ao público-alvo, e o fornecimento de ações de caráter preventivo a doenças e promovedoras da saúde, foram acrescidos dos princípios da Educação em Saúde, que consiste na integração dos saberes 
(científico e popular), dando possibilidade aos sujeitos envolvidos uma visão crítica e uma maior participação no processo de ensino-aprendizagem (GAZZINELLI; PENNA, 2006).

Nesse sentido, a exploração dos conteúdos expostos nas oficinas deu-se a partir do conhecimento prévio dos adolescentes, e visou esclarecer as transformações ocorridas em seus corpos nessa fase de desenvolvimento. Isso possibilita uma construção autônoma sobre os cuidados que podem adquirir a partir da implantação da educação em saúde.

Os adolescentes mostraram-se motivados pela busca de novas fontes de conhecimento, demonstrando, nas oficinas, um grande interesse pelos temas abordados.

Da mesma forma, considerando-se os objetivos do PCN (2000), tais como: o estudo dos sistemas e órgãos, prevenção de doenças, preservação da saúde coletiva e individual, riscos a integridade pessoal e social, valorização de hábitos saudáveis, métodos de contracepção, prevenção a doenças sexuais e gravidez indesejada; pode-se observar que, além do cumprimento das metas, alguns pontos puderam ser melhorados. Foi dada oportunidade aos alunos de se posicionarem criticamente em relação aos assuntos abordados, mostrando a eles a responsabilidade na escolha do que é saudável ou não para seu corpo.

Ao inserir-se o papel da Enfermagem nesse cenário morfológico, implantou-se uma nova maneira de disseminar o conhecimento, prevenir as doenças e promover a saúde por meio da educação continuada, ferramenta que promove o desenvolvimento das pessoas e lhes assegura a qualidade de vida (GRECO, 2004).

Assim, implantando-se a Educação em Saúde aos específicos sistemas corporais pode-se expor: no sistema ósseo, foi citada a osteoporose (hipocalcemia corporal), doença decorrente de uma perda significante de massa óssea, deixando os ossos porosos e, por conseguinte, susceptíveis a fraturas (SMELTZER; BARE, 2006). Ressaltou-se aos adolescentes que dentre os fatores de risco, causadores da perda de densidade óssea, estão o baixo consumo de derivados lácteos, a idade avançada e o consumo diário de bebidas alcoólicas, e que, desempenhando função protetora, têm-se, como exemplo, a atividade física diária por mais de 30 minutos e o consumo de alimentos ricos em cálcio (SILVA; MALINA, 2000).

Já na abordagem do sistema muscular, foi salientado que as contusões musculares e o estiramento muscular são causados por traumatismo direto, rápido e com aplicação de força compressiva sobre o tecido muscular ou quando o alongamento causado no músculo ultrapassa sua capacidade elástica, respectivamente. Qualquer que seja o mecanismo que cause a lesão, sempre haverá dor associada, o que intervirá na locomoção, no desempenho de atividades esportivas e da vida diária (FALCAI et al., 2010). Ainda, estudos indicam que as lesões musculares são comumente decorrentes de práticas esportivas e, tendo-se em vista que grande parte dos adolescentes possui uma vida esportiva ativa, foi reforçada a atenção e os cuidados que os mesmos devem ter durante as práticas esportivas (ALVES et al., 2005). Dentre as ações preventivas, compreendem-se as atividades de alongamento e de fortalecimento muscular, treinamento aeróbio, relaxamento e repouso pós-exercício e correção postural, orientados e conduzidos por profissionais integrantes de uma equipe multidisciplinar (RUSCHEL; MENEZES; HAUPENTHAL, 2009). 
Adicionalmente, quando abordada a Educação em Saúde junto ao sistema respiratório, focou-se a influência do tabagismo e a predisposição de doenças decorrentes do fumo. Dentre elas, as doenças respiratórias, câncer de traqueia/brônquios/pulmão, doença pulmonar obstrutiva crônica (DPOC) e acidentes vasculares encefálicos e cardíacos (OLIVEIRA; VALENTE; LEITE, 2008; SANTOS, 2009). Assim, foi exposta aos adolescentes a importância do aspecto preventivo, uma vez que o período da adolescência constitui a época de maior vulnerabilidade ao tabagismo (MALCON, 2003) e que a eliminação total dessa prática está relacionada à prevenção de $71 \%$ dos casos de câncer de pulmão e de 86\% de câncer de laringe (MENEZES; HORTA; OLIVEIRA, 2002).

Ainda, durante o enfoque do sistema cardiovascular, falou-se sobre o infarto agudo do miocárdio (IAM). Foi explicado aos adolescentes o que esta patologia e os motivos causadores, como a isquemia causada por arteriosclerose, ruptura súbita de vaso por aterosclerose e a formação de trombos, os quais podem causar obstrução do fluxo sanguíneo nos vasos cardíacos (PESARO; JUNIOR; NICOLAU, 2004; ALVES; FRÁGUAS; WAJNGARTEN, 2009). Nesse sentido, foram enfatizados aos estudantes os fatores de risco que desencadeiam esta condição patológica, sendo eles: obesidade, hipertensão arterial sistêmica (HAS), o tabagismo, a dislipidemia, diabetes mellitus (DM) e a falta de regularidade ou a prática de exercícios físicos (PALMA, 2008).

Ainda, os materiais amostrados dos sistemas reprodutor feminino e masculino, acrescidos da Educação em Saúde, levaram os educandos a falar sobre o uso de preservativos nas relações sexuais, bem como as possíveis consequências do ato sexual sem o mesmo: gravidez e doenças sexualmente transmissíveis (DST). Foi enfatizado que o uso de preservativos não se abstém somente à barreira contraceptiva, mas também como forma de proteção às doenças sexualmente transmissíveis (CARRENO; COSTA, 2006). Foi feita, também, uma correlação entre a adolescência, o consumo de drogas e a sexualidade, mostrando aos sujeitos da pesquisa uma associação entre o consumo de substâncias psicoativas e o uso não consciente de preservativos, o que pode levar à aquisição de DST's ou gravidez precoce (BERTONI et al., 2009).

Embora haja divulgação, por meio da mídia, dos Programas de Saúde Pública e de Prevenção, cujo intuito é disseminar a importância do uso do preservativo e seus efeitos positivos, percebe-se uma não eficiência, haja vista a defasagem de conhecimento mostrada pelos adolescentes que, somada à sensação de invulnerabilidade a qualquer doença, torna os jovens ainda mais expostos ao contágio das DST e AIDS (OLIVEIRA et al., 2009). Desta forma, a oportunidade deles aprofundarem o conhecimento a respeito dos sistemas reprodutores evidencia a ideia de facilidade e reais chances de transmissão de doenças, o que diminui a sensação de invulnerabilidade e aumenta a responsabilidade quanto ao uso de métodos contraceptivos.

A partir das informações geradas durante nosso trabalho, observou-se que alguns dos objetivos do PCN foram alcançados, tais como: conhecer e adotar hábitos de sexo seguro, conhecer e prevenir os meios de transmissão de doenças sexualmente transmissíveis, conhecer o seu próprio corpo, valorizar e cuidar de sua saúde como condição necessária para usufruir de prazer sexual; proteger-se de relacionamentos sexuais coercitivos ou exploradores; reconhecer o consentimento mútuo como necessário para usufruir de prazer numa relação a dois (BRASIL, 2000).

Em relação ao sistema nervoso, quando mostrado o feto com anencefalia, foi feita a 
associação da importância do uso do Acido Fólico na gravidez. O ácido fólico tem um papel fundamental no processo da multiplicação celular, sendo, portanto, imprescindível durante a gravidez. Sua ausência nesta fase de desenvolvimento corresponde a uma redução no fator de risco para desencadear defeitos do tubo neural em 50 a 70\% (PACHECO et al., 2009).

Durante o andamento do projeto, observou-se que a curiosidade dos adolescentes para com os sistemas morfológicos impulsionou o interesse deles para o conhecimento de danos ao corpo humano, bem como, às ações preventivas. Acredita-se que atividades educativas, trabalhadas nesta fase do desenvolvimento em que se encontra o público-alvo do projeto, possam trazer benefícios futuros a sua saúde.

\section{CONSIDERAÇÕES FINAIS}

Baseando-se nos resultados do projeto, acredita-se que a correlação entre a educação em saúde e a exploração do corpo humano trouxe benefícios para os sujeitos da pesquisa, uma vez que, conhecendo melhor seu próprio corpo, ações de prevenção às doenças e promoção à saúde podem ser mais rotineiras e eficazes.

Da mesma forma, a junção do ensino teórico às aulas práticas facilitou o entendimento, pelos alunos, dos sistemas expostos. Ademais, a exposição de doenças e má formação nas peças cadavéricas minimizou a ideia, típica da idade, de não vulnerabilidade, maximizando a ideia de que as doenças realmente acontecem e podem manifestar-se em qualquer ser humano.

\section{REFERÊNCIAS}

ALVES, J. G. B. et al. Prática de esportes durante a adolescência e atividade física de lazer na vida adulta. Rev. Bras. Med. Esporte, São Paulo, v. 11, n. 5, set.-out. 2005.

ALVES, T. C. T. F.; FRÁGUAS, R.; WAJNGARTEN, M. Depressão e infarto agudo do miocárdio. Rev. Psiquiatr. Clín., São Paulo, v. 36, supl. 3, 2009.

BERTONI, N. et al. Uso de álcool e drogas e sua influência sobre as práticas sexuais de adolescentes de Minas Gerais, Brasil. Cad. Saúde Pública, Rio de Janeiro, v. 25, n. 6, jun. 2009.

BRASIL. Ministério da Educação. Secretaria de Educação Fundamental. Parâmetros Curriculares Nacionais: pluralidade cultural: orientação sexual. Rio de Janeiro: DP\&A, 2000.

CANALI, E. S.; KRUEL, L. F. M. Respostas hormonais ao exercício. Rev. Paul. Educ. Fís., São Paulo, v. 15, n. 2, p. 141-153, jul./dez. 2001.

CARRENO, I; COSTA, J. S. D. Uso de preservativos nas relações sexuais: estudo de base populacional. Rev. Saúde Pública, São Paulo, v. 40, n. 4, ago. 2006. 
DÂNGELO, J. G; FATTINI, C. A. Anatomia humana sistêmica e segmentar: para o estudante de medicina. 2. ed. São Paulo: Atheneu, 2002.

FALCAI, M.J. et al. Análise biomecânica e histológica de músculos gastrocnêmios de ratas submetidas à lesão muscular e tratados com laserterapia de baixa intensidade. Rev. Bras. Ortop., São Paulo, v. 45, n. 4, 2010.

FAUSTINI, D. M. T. et al. Programa de orientação desenvolvido com adolescentes em centro de saúde: conhecimentos adquiridos sobre os temas abordados por uma equipe multidisciplinar. Ciência \& Saúde Coletiva, Rio de Janeiro, v. 8, n. 3, p. 783-790, 2003.

FERREIRA, M. A. F. Trabalho infantil e produção acadêmica nos anos 90: tópicos para reflexão. Estudos de Psicologia, Natal, v. 6, n. 2, p. 213-225, 2001.

FREITAS K. R.; DIAS, S. M. Z. Percepções de adolescentes sobre sua sexualidade. Texto Contexto Enferm., Florianópolis, v. 19, n. 2, abr.-jun. 2010.

GAZZINELLI, M. F.; PENNA, C. Educação em Saúde: conhecimentos, representações sociais e experiência da doença. In: Educação em Saúde: teoria, método e imaginação. Belo Horizonte: UFMG, 2006.

GOMES, W. A. et al. Nível de informação sobre adolescência, puberdade e sexualidade entre adolescentes. J. Pediatr., Rio Janeiro, v. 78, n. 4, p. 301-308, 2002.

GRECO, R. M. Relato de experiência: ensinando a administração em enfermagem através da educação em saúde. Rev. Bras. Enferm., Brasília, v. 57, n. 4. jul.-ago. 2004.

MALCON, M. C. et al. Prevalência e fatores de risco para tabagismo em adolescentes na América do Sul: uma revisão sistemática da literatura. Rev Panam. Salud Publica, v.13, n. 4, p. 222-228, 2003.

MENEZES, A. M. B.; HORTA, B. L.; OLIVEIRA, A. L. B. Risco de câncer de pulmão, laringe e esôfago atribuível ao fumo. Rev. Saúde Pública. São Paulo, v. 36, n. 2, abr. 2002.

MONTES, M. A. A.; CARDOSO, V. T. S.; SOUZA, C. T. V. Popularização da ciência e da arte através da Anatomia Humana. In: REUNIÃO DA REDE DE POPULARIZAÇÃO DA CIÊNCIA/ CONGRESSO MUNDIAL DE MUSEUS DE CIÊNCIAS, 9., 2005, Rio de Janeiro. Anais... Rio de Janeiro, 2005, p. 53.

OLIVEIRA, A. F.; VALENTE, J. G.; LEITE, I. C. Aspectos da mortalidade atribuível ao tabaco: revisão sistemática. Rev. Saúde Pública, São Paulo, v. 42, n. 2, abr. 2008.

OLIVEIRA, D. C. et al. Conhecimentos e práticas de adolescentes acerca das DST/HIV/AIDS em duas escolas públicas municipais do Rio de Janeiro. Esc. Anna Nery. Revista de Enfermagem, Rio de Janeiro, v. 13, n. 4, out.-dez. 2009.

PACHECO, S. S. et al. Efeito da fortificação alimentar com ácido fólico na prevalência de defeitos do tubo neural. Rev. Saúde Pública, São Paulo, v. 43, n. 4, ago. 2009. 
PALMA, A. Reflexões sobre o "sedentarismo" na pesquisa sobre fatores de risco para infarto agudo do miocárdio. Rev Panam. Salud Publica, Washington, v. 24, n. 5, nov. 2008.

PESARO, A. E. P.; JUNIOR, C. V. S.; NICOLAU, J. C. Infarto agudo do miocárdio - síndrome coronariana aguda com supradesnível do segmento ST. Rev. Assoc. Med. Bras., São Paulo, v. 50, n. 2, abr.-jun. 2004.

RUSCHEL, C., MENEZES, F.S., HAUPENTHAL, A. Incidência de lesões em velejadores brasileiros de diferentes níveis técnicos. Rev. Bras. Med. Esporte, Niterói, v. 15, n. 4, jul.-ago. 2009.

SANTANA, M. T. B. M.; JORGE, M. S. B. The own body as a nurse's living experience on assisting others in their dyng process. Rev. Latino-Am. Enfermagem, Ribeirão Preto, v. 15, n. 3, jun. 2007.

SANTOS, U. P. Cessação de tabagismo: desafios a serem enfrentados. Rev. Assoc. Med. Bras., São Paulo, v. 55, n. 5, 2009.

SILVA, C. C. et al. O exercício físico potencializa ou compromete o crescimento longitudinal de crianças e adolescentes? Mito ou verdade? Rev. Bras. Med. Esporte., Niterói, v. 10, n. 6, dez. 2004.

SILVA, R. C. R.; MALINA, R. M. Nível de atividade física em adolescentes do Município de Niterói, Rio de Janeiro, Brasil. Cad. Saúde Pública, Rio de Janeiro, v.16, n.4, out./dez. 2000.

SMELTZER, S. C.; BARE, B. G. Tratado de Enfermagem Médico-Cirúrgica. 8. ed. São Paulo: Guanabara Koogan, 2006.

VAN DE GRAAF, K. M. Anatomia Humana. 6. ed. São Paulo: Manole, 2003.

Submetido em $1^{\circ}$ de março de 2012.

Aprovado em $1^{\circ}$ de junho de 2012. 


\section{ANEXO I}

\section{QUESTIONÁRIO (SANTANA; JORGE, 2007).}

1. Você entende o que é a Anatomia Humana?

( ) Sim, entendo. ( ) Não muito. ( ) Não, não entendo.

2. Você já viu algum material cadáverico?

( ) Sim. ( ) Não.

3. Você já visitou algum Laboratório de Anatomia Humana?

( ) Sim. ( ) Não.

4. Você acha importante o conhecimento do Corpo Humano?

( ) Sim. ( ) Não.

5. Você sabe identificar o que é fisiológico (normal) no seu próprio corpo?

( ) Sim. ( ) Não.

6. Qual dos sistemas abaixo você mais se interessa em explorar no Laboratório de Anatomia Humana?

( ) Sistema Ósseo.

( ) Sistema Articular.

( ) Sistema Muscular.

( ) Sistema Cardíaco.

( ) Sistema Respiratório.

( ) Sistema Digestório.

( ) Sistema Urinário.

( ) Sistema Reprodutor Feminino e Masculino.

( ) Sistema Nervoso.

7. Você sabe quais as mudanças que ocorrem no seu corpo na fase da Puberdade?

( ) Sim. ( ) Não.

8. Você acha que esta pesquisa te acrescentará algo?

( ) Sim. ( ) Não. 


\section{ANEXO II}

\section{Cronograma de Execução e Plano de Trabalho}

\begin{tabular}{|c|c|c|}
\hline ETAPAS & DATA & DURAÇÃO \\
\hline Primeiro contato com a direção da escola. & 17.01.2011 & 50 minutos \\
\hline Segundo contato com a direção da escola. & 10.02 .2011 & 40 minutos \\
\hline $\begin{array}{l}\text { Agendamento com a direção da escola para a reunião } \\
\text { com os pais dos alunos para exposição dos objetivos do } \\
\text { estudo. }\end{array}$ & 18.03 .2011 & $\begin{array}{l}\text { Diretor do Colégio não } \\
\text { compareceu, pois estava } \\
\text { em uma reunião em BH. }\end{array}$ \\
\hline $\begin{array}{l}\text { Agendamento com a direção da Escola para a reunião } \\
\text { com os pais dos alunos para exposição dos objetivos do } \\
\text { estudo, e agendamento da apresentação do estudo para } \\
\text { os alunos. }\end{array}$ & 25.03.2011 & 60 minutos \\
\hline $\begin{array}{l}\text { Primeiro contato com os alunos, apresentação do estudo } \\
\text { e dos profissionais atuantes. Distribuição da convocação } \\
\text { para a reunião e autorização dos pais. }\end{array}$ & 30.03 .2011 & 90 minutos \\
\hline Reunião com os pais dos alunos na escola em estudo. & 07.04 .2011 & 20 minutos \\
\hline $\begin{array}{l}\text { Primeiro dia de Oficina - Aula Teórica } \\
\text { Discussão e Preenchimento do Questionário } 01 . \\
\text { "O que é a Anatomia Humana? De onde ela surgiu?", } \\
\text { "O que é o corpo para você?" } \\
\text { "Qual parte do corpo você mais se interessa?" } \\
\text { "Você já viu um corpo sem vida?" } \\
\text { "Você gostaria de ver um corpo sem vida?" }\end{array}$ & 31.04 .2011 & 240 minutos \\
\hline $\begin{array}{l}\text { Segundo dia de Oficina - Aula Teórico-Prática } \\
\text { do Aparelho Locomotor: "Sistema Esquelético, Sistema } \\
\text { Articular e Sistema muscular". }\end{array}$ & 02.05 .2011 & 240 minutos \\
\hline $\begin{array}{l}\text { Terceiro dia de Oficina - Aula Teórico-Prática de } \\
\text { Esplancnologia: "Sistema Cardiovacular, Sistema } \\
\text { Respiratório, Sistema Digestório". }\end{array}$ & 03.05 .2011 & 240 minutos \\
\hline $\begin{array}{l}\text { Quarto dia de Oficina - Aula Teórico-Prática de } \\
\text { Aparelho Urogenital e Neuroanatomia: } \\
\text { "Sistema Reprodutor Feminino e Masculino e Sistema } \\
\text { Nervoso". }\end{array}$ & 04.05 .2011 & 240 minutos \\
\hline
\end{tabular}

